\title{
Exotic Decay Modes in Rotating Nuclei
}

\author{
D. Rudolph ${ }^{\text {a }}$, E.K. Johansson ${ }^{\text {a }}$, L.-L. Andersson ${ }^{\text {a }}$, J. Ekman ${ }^{\text {a,b }}$, C. Fahlander ${ }^{\text {a }}$, and \\ R. du Rietz ${ }^{\text {a }}$
}

${ }^{a}$ Department of Physics, Lund University, S-22100 Lund, Sweden

bTechnology and Society, Malmö University, S-20506 Malmö, Sweden

Recent results and experiments are presented aiming at nuclear structure studies in the vicinity of the doubly-magic isotope ${ }^{56} \mathrm{Ni}$. The feature of prompt particle emission is revised, and new studies of mirror nuclei in the upper $f p$ shell are briefly discussed.

\section{INTRODUCTION}

Doubly-magic nuclei are important bench marks within the nuclidic chart. These nuclei and their nearby neighbours serve as sources and act as constraints for the parameter sets used to describe the atomic nucleus by means of the nuclear shell-model. This contribution focuses on recent advances in high-spin nuclear structure studies in the vicinity of ${ }^{56} \mathrm{Ni}$. This isotope is generally accepted to represent a doubly-magic spherical nucleus due to the shell gap at particle number 28 , which separates the $1 f_{7 / 2}$ shell from the so-called upper $f p$ shell consisting of the $2 p_{3 / 2}, 1 f_{5 / 2}$, and $2 p_{1 / 2}$ orbits.

In proton-rich nuclei in the vicinity of ${ }^{56} \mathrm{Ni}$ a new nuclear decay mode called prompt particle emission has been established during recent years. The first case of prompt proton emission from excited high-spin states in the well-deformed second minimum of the nuclear potential into spherical daughter states was identified in ${ }^{58} \mathrm{Cu}[1]$. In the following years, additional prompt proton decays have been found in ${ }^{56} \mathrm{Ni}[2]$ and ${ }^{59} \mathrm{Cu}[3]$, and a case of prompt $\alpha$-particle emission has been observed in ${ }^{58} \mathrm{Ni}[4]$.

The decay mode is called 'prompt', because the formation of the residual nucleus, the $\gamma$-decay of the rotational band in the second minimum, the particle emission, and finally the $\gamma$ decay in the daughter nucleus are observed in 'prompt' coincidence in thin-target inbeam fusion-evaporation experiments. The particle decays compete with the conventional $\gamma$ decay-out, which places the time scale of the decays into the $10^{-12}-10^{-15} \mathrm{~s}$ regime. They may be viewed as self-regulated two-dimensional quantum tunneling processes: The problem is two-dimensional, because the initial states have prolate deformation with a long-to-short axis ratio of about 1.5:1 (see, e.g., Refs. $[1,5]$ ). The process is self-regulated, because the emission of the particles induces a drastic rearrangement of the nuclear meanfield potential, since the final states are spherical or even slightly oblate deformed. In fact, the shape change associated with the decay mode seems to cause major problems in the theoretical description of the process, though first suggestions have been put forward recently in the case of ${ }^{58} \mathrm{Cu}[6]$. 


\section{PROMPT PARTICLE DECAYS FROM ROTATIONAL BANDS}

\subsection{Summary of results}

The experimental knowledge concerning the prompt proton decays has been increased systematically following the first observation of the decay mode [1] at GAMMASPHERE [7]. The focus has been on ${ }^{58} \mathrm{Cu}$, where spins and parities of the initial and final state could be assigned $[5,8,9]$ and lifetimes of $\gamma$ - and proton-decaying states have been measured in the decay-out regime of the rotational band $[5,10]$.

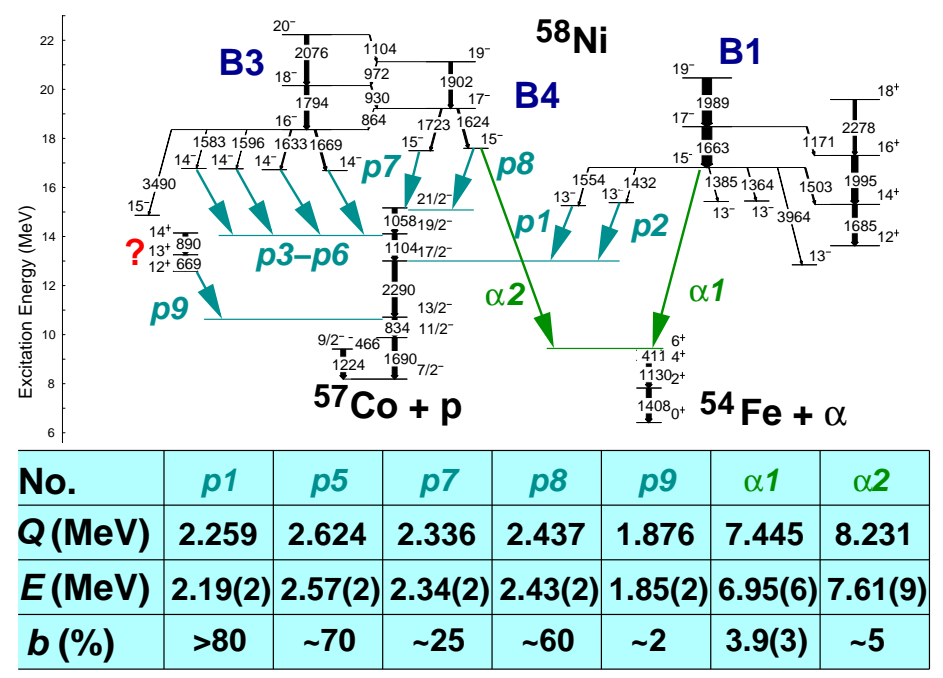

Figure 1. Particle decay scheme (preliminary) of high-spin deformed and superdeformed bands in ${ }^{58} \mathrm{Ni}$ [13]. A total of nine proton and two $\alpha$-decays are observed. The scheme is embedded in a high-spin decay scheme of ${ }^{58} \mathrm{Ni}$ comprising more than $300 \gamma$-ray transitions reaching spin $I=32$ at some $40 \mathrm{MeV}$ excitation energy.

A first dedicated experiment aiming at in-beam high-resolution particle- $\gamma$ coincidence spectroscopy has been performed in December 1998 at Argonne National Laboratory using GAmmASPHERE [7], a wall of four LASSA-type $\Delta E$-E Si-strip telescopes [11] at forward angles, rings 4-9 of MicrobalL [12], and 20 neutron detectors ('UPenn-UK'-array). A refined study of the prompt proton decay in ${ }^{58} \mathrm{Cu}$ was feasible [9], and the first case of 'fine structure' was observed in ${ }^{59} \mathrm{Cu}[3]$. Subsequently, ${ }^{58} \mathrm{Ni}$ has been revisited, and Fig. 1 provides a preliminary 'particle decay scheme' of normally and superdeformed states of that nucleus into ${ }^{57} \mathrm{Co}$ and ${ }^{54} \mathrm{Fe}$, respectively. The total of nine proton decay lines and two $\alpha$ decay lines in one isotope marks the next mile stone in the study of this decay mode [13]. One state decays via proton $(p 8), \alpha-(\alpha 2)$, and $\gamma$ emission (not resolved). Another exceptional case is the proton branch $p 9$, which proceeds from a presumed weakly deformed state. Given the spin and parity assignments, which are fixed through numerous parallel 
$\gamma$-decay branches, it represents the first $1 h_{11 / 2}$ proton decay - all other cases known so far are $1 g_{9 / 2}$ protons. The rather small proton $p 9$ branching ratio of some $2 \%$ is also very different from the 'normal' proton decays, and it appears possible to probe the presence of small superintruder $\mathcal{N}=5 h_{11 / 2}$ components in the wave function of $A \sim 60$ nuclei by means of combined particle- $\gamma$ spectroscopy. The full high-spin level scheme of ${ }^{58} \mathrm{Ni}$ comprises more than $300 \gamma$-ray transitions reaching spin $I=32$ at a world-record excitation energy of $40 \mathrm{MeV}[13]$.

Figure 2 shows preliminary results of the proton angular distribution (decay $p 8$ in ${ }^{58} \mathrm{Ni}$ ) relative to the spin axis of emitting ${ }^{58} \mathrm{Ni}$ nuclei from the previous experiment. Only a subset of data is used where the evaporated $\alpha$-particle and the later-on emitted proton were both detected in one of the four Si-strip telescopes. The overall shape of the distribution is consistent with the expectations for a particle with rather high angular momentum $[14,15]$. Interestingly, the peak around $90^{\circ}$ indicates an emission from the tips of the axially deformed ${ }^{58} \mathrm{Ni}$ nucleus, which has been suggested as early as 1953 [16] (for $\alpha$-decay from deformed nuclei). It remains to be seen (see below) whether eventual deviations from the basic shape of the angular distribution may provide access to more fundamental issues such as the radial distribution of a $1 g_{9 / 2}$ proton in a deformed nuclear mean field or the exploration of the nuclear time scale of the shape change of the nucleus $[17,18]$.

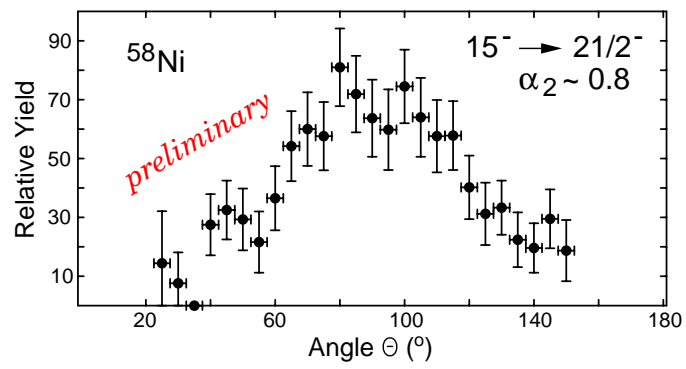

Figure 2. Angular distribution relative to the spin axis for one of the $1 g_{9 / 2}$ prompt proton decays in ${ }^{58} \mathrm{Ni}$ (preliminary). See text for details.

\subsection{New experiments}

Future investigations of the prompt particle decays in this mass region shall focus on the angular distributions and correlations of the emitted particles, preferably with respect to the spin axis of the emitting residue. Usually, measurements in nuclear physics produce quantities, i.e., expectation values or matrix elements, which are averaged over coordinates or time. Different from that, the angular distribution of single nucleons is directly related to the spatial distribution of the single-particle wave function [17]. In addition, a precise determination of the particle angular distribution may also reveal a glimpse of the nuclear time scale. It should be noted that the spin alignment coefficient is of the order of $\alpha_{2} \sim 0.7-0.8$ when the particles are emitted from the high-spin states. In this case the spin alignment is a natural consequence of the reaction mechanism, while proton angular distribution measurements of ground-state- or $\beta$-delayed proton emitters 
require low temperature hyperfine interaction techniques $[19,20]$.

To address the measurement of angular distributions of the protons and $\alpha$ particles associated with prompt particles, an experiment was performed in April 2004 at the ATLAS facility at Argonne National Laboratory. The set-up is sketched in Fig. 3. A newly developed array of eight $\Delta E-E$ Si-strip telescopes, named LuWuSiA, was placed around the reaction target together with the two most backward rings of the CsI array MicrobaLL. Each of the $\Delta E$ and $E$ detectors has a 16-strip read-out, and their combination gives rise to some 1800 active pixels between $\sim 10^{\circ}<\theta<\sim 120^{\circ}$ relative to the beam axis. The $\mathrm{Si}$ telescopes replaced $80 \mathrm{CsI}$ detector elements of MiCROBALL.

This charged-particle arrangement was placed inside the GAMmAsPHERE array, which comprised 77 Ge-detectors at the time of the experiment. 30 neutron detectors replaced the same number of Ge-detectors at forward angles, and this NEuTron SHELL [21] served (together with the Fragment Mass Analyzer [22]) to separate the large number of reaction channels.

Next to the above discussed prime objective of charged particle angular distributions, a plethora of secondary issues will be explored with this complex data set:

- The search for new prompt particle decays in the mass region. Based on Fig. 1 and Ref. [3] one can state that prompt particle decays appear to be a common feature of at least this mass regime. Increased statistics and improved resolution will allow for a better discrimination of them.

- The identification or confirmation of excited states in exotic reaction channels having $N<Z$, e.g., ${ }^{61,62} \mathrm{Ge}(3 n, 2 n),{ }^{61} \mathrm{Ga}(1 p 2 n)$, and ${ }^{58} \mathrm{Zn}(1 \alpha 2 n)$.

- Particle decaying normally deformed or spherical states (cf. ${ }^{58} \mathrm{Ni}$ above), in particular in the $N<Z$ nuclei mentioned above. Such states are seen, e.g., in ${ }^{57} \mathrm{Cu}$ following the $\beta$ decay of ${ }^{57} \mathrm{Zn}$ [23], and presumed to exist in ${ }^{61} \mathrm{Ga}$ [24] (see below).

- HIGH-spin spectroscopy of the two-particle evaporation channels ${ }^{62} \mathrm{Zn}(2 p)$ and ${ }^{62} \mathrm{Ga}$ $(1 p 1 n)$. If these nuclei are produced in reactions optimized for four-particle emission, they are created at very high spins and excitation energies. Recent results in ${ }^{58} \mathrm{Ni}$ (when produced via $2 p$ evaporation) and a preliminary analysis of ${ }^{62} \mathrm{Zn}$ indicate $\gamma$-ray transitions of up to $4.4 \mathrm{MeV}$ on top of the superdeformed bands at excitation energies of about $40 \mathrm{MeV}$. This converts into rotational frequencies of $\hbar \omega=2.2 \mathrm{MeV}$, where the influence of the $\mathcal{N}=51 h_{11 / 2}$ super-intruder orbital may drive the nucleus to even larger deformations. Cranked Nilsson-Strutinsky calculations indicate that such bands become yrast at spin $I \sim 30-32$ or $\hbar \omega \sim 1.8 \mathrm{MeV}$, respectively [25]. Of course, (some of) these bands may particle decay.

- 'Complete' spectroscopy of strongly populated reaction channels.

In the near future we intend to perform an in-beam experiment dedicated to ${ }^{57} \mathrm{Cu}$, in which a combination of two CD-type double-sided Si-strip (DSSD) detectors and the (almost) complete MicroBALL shall be explored. Besides the possibility of particle tracking and proper position determination for low-energy particles, the set-up foresees DSSD-CsI and DSSD-DSSD-CsI telecopes. Such combinations are considered good candidates for high-efficient and high-precision charged-particle arrays for the next generation of Gedetector arrays, such as the AgATA demonstrator or GRETinA. 


\section{GSFMA $138 \quad(04 / 2004)$}

\section{${ }^{36} \mathrm{Ar}+{ }^{28} \mathrm{Si}$ @ $142 \mathrm{MeV}$ \\ Aim: Particle spectroscopy II}

Argonne National Laboratory
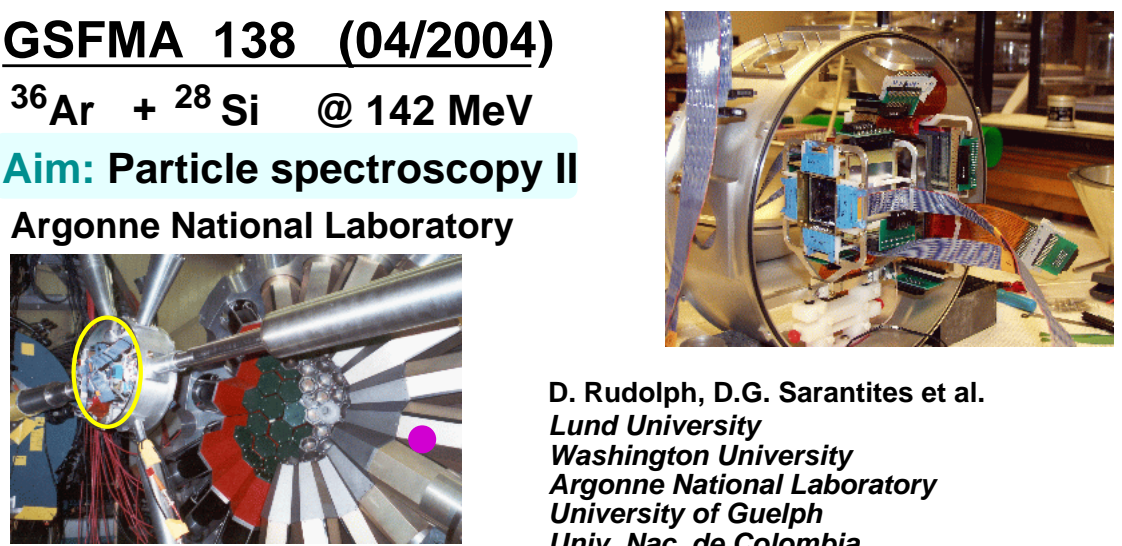

D. Rudolph, D.G. Sarantites et al.

Lund University

Washington University

Argonne National Laboratory

University of Guelph

Univ. Nac. de Colombia

- GAMMASPHERE (77 detectors)

Thin target $\left(0.2 \mathrm{mg} / \mathrm{cm}^{2}\right)$

16 MICROBALL CsI

8 Si-strip telescopes (256 channels)

Neutron Shell (30 detectors)

- Fragment Mass Analyzer

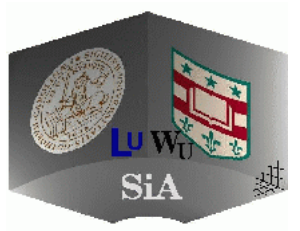

Figure 3. Parameters of the most recent experiment to study prompt particle decays in the mass $A=60$ region.

\section{PROMPT PARTICLE DECAYS FROM NEAR SPHERICAL STATES - MIRROR NUCLEI IN THE UPPER $f \boldsymbol{p}$ SHELL}

During the past decade the experimental knowledge on $T=1 / 2$ and $T=1$ mirror nuclei in the $1 f_{7 / 2}$ shell has increased substantially (see, e.g., Refs. [26-32]), and this progress has been accompanied with detailed theoretical studies and refinements, for example, in Refs. $[28,30,31,33]$. Close to the center of the $1 f_{7 / 2}$ shell the observed mirror energy differences (MED) - the differences between level energies of adjacent states in pairs of mirror nuclei - are typically $10-100 \mathrm{keV}$, and they are (most often) readily explained by several Coulomb monopole and multipole effects. On the contrary, differences in configurations of adjacent states may cause significant changes in Coulomb monopole contributions in nuclei below and above the rather well isolated $1 f_{7 / 2}$ shell. One such example is the $\sim 300 \mathrm{keV}$ drop in MED between the $11 / 2^{-}$and $13 / 2^{-}$states in the $A=35$ and $A=39$ mirror systems, which can be explained by radial effects as well as a hitherto overlooked electromagnetic spin-orbit contribution [34].

To extend the experimental knowledge into the upper $f p$ shell, an experiment was conducted at the Holifield Radioactive Ion Beam Facility at Oak Ridge National Laboratory to identify excited states in ${ }^{61} \mathrm{Ga}$ and ${ }^{62} \mathrm{Ge}$. In fusion-evaporation reactions of a ${ }^{40} \mathrm{Ca}$ beam at $104 \mathrm{MeV}$, impinging on a $99.92 \%$ isotropically enriched ${ }^{24} \mathrm{Mg}$ target foil of thickness 
$0.3 \mathrm{mg} / \mathrm{cm}^{2},{ }^{64} \mathrm{Ge}$ compound nuclei are formed. The ${ }_{31}^{61} \mathrm{Ga}_{30}$ nuclei are then produced via the $1 p 2 n$ reaction channel and ${ }_{32}^{62} \mathrm{Ge}_{30}$ following the evaporation of two neutrons.

The Ge detector array CLARION [35] was used to detect the $\gamma$ radiation at the target position in coincidence with the recoiling nuclei, which were discriminated with the help of the recoil mass spectrometer (RMS) before finally being stopped in an Ionisation Chamber (IC). The horizontal position, i.e., the $A / Q$ value, is determined by a position-sensitive grid placed inside the IC [35], and event-by-event information on the proton number $Z$ was obtained through detailed investigations of differential energy losses in the three parts of the anode in the IC [36].

A total of five transitions were found to belong to ${ }^{61} \mathrm{Ga}[36,24]$. Recoil- $\gamma \gamma$ coincidences and mirror symmetry arguments suggest that three transitions at 1506, 1126, and $271 \mathrm{keV}$ form the $13 / 2^{-} \rightarrow 9 / 2^{-} \rightarrow 5 / 2^{-} \rightarrow 3 / 2^{-}$cascade in ${ }^{61} \mathrm{Ga}$. The mirror transitions in ${ }^{61} \mathrm{Zn}$ have energies of 1532,1141 , and $124 \mathrm{keV}$, respectively [37,38]. A $220 \mathrm{keV}$ transition in ${ }^{61} \mathrm{Ga}$ finds a natural counterpart in the $89 \mathrm{keV} 1 / 2^{-} \rightarrow 3 / 2^{-}$ground-state transition in ${ }^{61} \mathrm{Zn}$ [39], but no unique candidate is at hand for the fifth line at $1231 \mathrm{keV}$. The level schemes of the $T_{z}= \pm 1 / 2 A=61$ mirror nuclei are shown in Fig. 4(a).

(a)

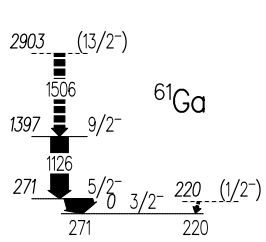

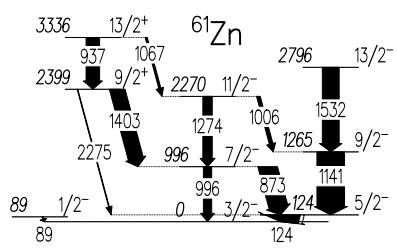

(b)
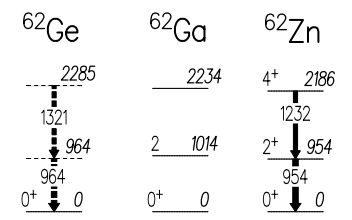

Figure 4. The proposed level schemes of ${ }^{61} \mathrm{Ga}$ [part (a)] and ${ }^{62} \mathrm{Ge}[$ part(b)] together with the relevant parts of the known level scheme of the mirror nuclei ${ }^{61} \mathrm{Zn}[39,37,38]$ and ${ }^{62} \mathrm{Zn}$ [42] as well as the presumed $2^{+}$and $4^{+}$isobaric analogue states in the odd-odd $N=Z$ nucleus ${ }^{62} \mathrm{Ga}$ [41]. Energy labels are in $\mathrm{keV}$, tentative transitions and levels are dashed, and the widths of the arrows correspond to the relative intensities of the transitions.

Two tentative transitions could be discriminated for the exotic $N=Z-2$ nucleus ${ }^{62} \mathrm{Ge}$ at 964 and $1321 \mathrm{keV}$ via careful inspections of correlations between differential energy losses in the IC and associated $\gamma$ rays [40]. Figure 4(b) compares the tentative excitation scheme of ${ }^{62} \mathrm{Ge}$ to its isobars ${ }^{62} \mathrm{Ga}$ and ${ }^{62} \mathrm{Zn}[41,42]$. In Fig. 5 the energy-loss behaviour of the $964 \mathrm{keV}$ candidate is compared to the behaviour of other candidates [(part (a)] and the ground-state transitions of the $A=62$ isobars ${ }^{62} \mathrm{Ga}(571 \mathrm{keV})$ and ${ }^{62} \mathrm{Zn}(954 \mathrm{keV})$ [(part (b)]. There is a small though rather distinct peak at the correct position for $Z=32$ for the $964 \mathrm{keV}$ candidate, while the rise at channel $\sim 200$, i.e., the $Z=30$ position, may be due to either a weak doublet with a transition in ${ }^{62} \mathrm{Zn}$ or problems in the background subtraction [40]. None of the other candidates around 950-1000 keV reveals a similarly pronounced rise at $Z=32$. It is estimated that about twice the statistics of the present experiment would be needed to actually prove that the $964 \mathrm{keV}$ line represents the $2^{+} \rightarrow 0^{+}$ground-state transition of ${ }^{62} \mathrm{Ge}$. Note that the relative production cross 
sections of ${ }^{62} \mathrm{Ge}$ and ${ }^{62} \mathrm{Zn}$ amount to 1:5000 (based on the relative yields of the 964 and $954 \mathrm{keV}$ transitions), and that ${ }^{62} \mathrm{Zn}$ is certainly not one of the main reaction channels.
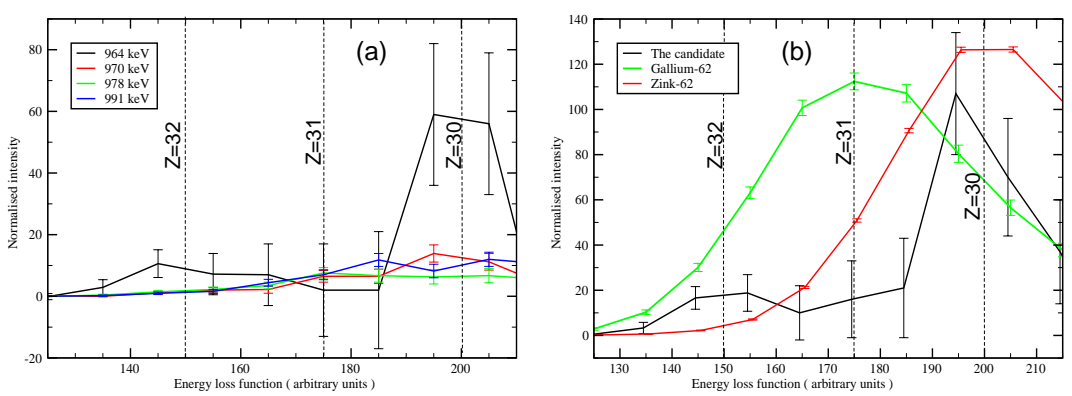

Figure 5. The energy-loss behaviour of different $A=62 \gamma$-ray transitions. The $x$-axis is a measure of the proton number, $Z$, and the expected positions for $Z=30,31$, and 32 are indicated by the dashed lines. In part (a) different candidates for being the ${ }^{62} \mathrm{Ge}$ ground-state transition are compared. The prime candidate at $964 \mathrm{keV}$ is compared to the energy loss behaviour of the ground state transitions in ${ }^{62} \mathrm{Ga}$ and ${ }^{62} \mathrm{Zn}$ in part (b).

The experimental MED values of the $A=61 T_{z}= \pm 1 / 2$ nuclei are illustrated in Fig. 6 . The most striking features are the $150 \mathrm{keV}$ energy difference between the $5 / 2^{-} \rightarrow 3 / 2^{-}$ ground state transitions in the two nuclei and, possibly, the $130 \mathrm{keV}$ difference between the presumed $1 / 2^{-} \rightarrow 3 / 2^{-}$transitions. In $f p$ shell nuclei relatively large energy differences like these have so far only been observed between low-lying single-particle states in the $A=41$ and $A=57$ mirror systems $[43,44]$ and between core excited states in the $A=51$ mirror pair [45].

These differences originate most likely from Coulomb monopole effects such as radial or electromagnetic spin-orbit contributions. The latter has only recently been introduced to explain MED values of up to $350 \mathrm{keV}$ in the mass $A=35$ and $A=39$ mirror pairs [34] and should come into play whenever single-nucleon excitations occur between $j=l+1 / 2$ orbitals (e.g., $2 p_{3 / 2}$ ) and $j=l-1 / 2$ orbitals (e.g., $1 f_{5 / 2}$ or $2 p_{1 / 2}$ ). Radial effects can be expected to play a major role when single-nucleon excitations between orbits of different angular momentum occur, since this implies a change in the spatial extent of the charge distribution in at least one of the mirror partners. Examples are $1 f_{7 / 2} \rightarrow 2 p_{3 / 2}$ or $2 p_{3 / 2} \rightarrow$ $1 f_{5 / 2}$ single-particle transitions in the $A=41$ and $A=57$ mirror systems, respectively.

Predictions from large-scale shell-model calculations using the shell-model code ANTOINE $[46,47]$ are included in Fig. 6. The calculations were performed in the full $f p$ space containing the $1 f_{7 / 2}$ orbit below and the $2 p_{3 / 2}, 1 f_{5 / 2}$, and $2 p_{1 / 2}$ orbits above the $N=Z=28$ shell closures. The configuration space was truncated to allow up to three particle excitations from the $1 f_{7 / 2}$ shell into the upper $f p$ shell. The calculations were performed using the KB3 with Coulomb interaction, where the two-proton matrix elements are constructed by adding harmonic oscillator Coulomb matrix elements to the bare two-body matrix elements. 
In the first calculation identical single-particle energies for protons and neutrons were used to estimate the Coulomb multipole component, $V_{C M}$, which takes the effect from the alignment of proton pairs into account. The result is shown as filled circles in Fig. 6. It is seen that the correct sign of the MED values is reproduced, although the calculated MED values are 50 to $100 \mathrm{keV}$ smaller than the experimental MED values. These discrepancies may be the result of Coulomb monopole effects, $V_{C m}$, which are not included in the calculation.

Calculations where the single-particle energies have been modified according to a contribution from the electromagnetic spin-orbit interaction, $V_{C l s},[34,36]$ is shown in Fig. 6 as filled squares. It is seen that the agreement with the observed MED values is nearly perfect, and there seems to be no need for other Coulomb monopole effects such as changes in nuclear radii or shapes.

The situation is somewhat less clear for the $A=62$ mirror pair ${ }^{62} \mathrm{Ge}$ and ${ }^{62} \mathrm{Zn}$. First of all, the experimental results are only tentative. Secondly, the predicted $2^{+}$energies are off by some $200 \mathrm{keV}$ on an absolute scale, which is probably due to the (until now necessary) exclusion of the $1 g_{9 / 2}$ orbital from the full $f p$ shell calculations. The inclusion of the $V_{C l s}$ contribution clearly helps to explain the positive MED value of the $4_{1}^{+}$state, but only half way. Interestingly, the experimental values lie in between the predictions of the yrast and yrare $2^{+}$and $4^{+}$states, i.e., agreement could be obtained if one considered the observed states as being a mixture of the predicted yrast and yrare levels.
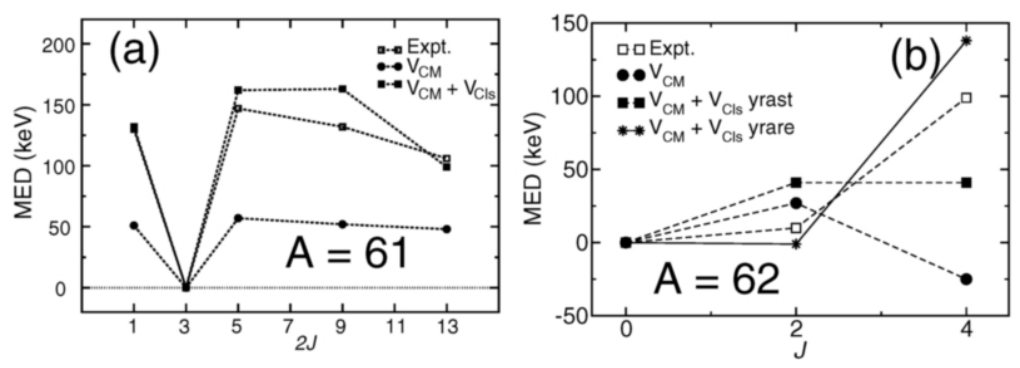

Figure 6. MED diagram of the $A=61$ (a) and $A=62$ (b) mirror nuclei. Open squares indicate experimental data, filled circles indicate calculated MED values including the $V_{C M}$ component, and filled squares indicate MED values including the $V_{C M}+V_{C l s}$ component.

Last but not least it is intriguing to take a closer look at the level schemes in Fig. 4. There is no apparent hint for the $9 / 2^{+} \rightarrow 7 / 2^{-} \rightarrow 5 / 2^{-}\left(1403-873 \mathrm{keV}\right.$ in $\left.{ }^{61} \mathrm{Zn}\right)$ or the $9 / 2^{+} \rightarrow 7 / 2^{-} \rightarrow 3 / 2^{-}\left(1403-996 \mathrm{keV}\right.$ in $\left.{ }^{61} \mathrm{Zn}\right)$ sequence in ${ }^{61} \mathrm{Ga}$ in the present data set, even though the branch through the $2399 \mathrm{keV} 9 / 2^{+}$state in ${ }^{61} \mathrm{Zn}$ has about the same intensity as the $13 / 2^{-} \rightarrow 9 / 2^{-} \rightarrow 5 / 2^{-}$cascade. A possible explanation for the nonobservation of $\gamma$ rays decaying from a $9 / 2^{+}$state in ${ }^{61} \mathrm{Ga}$ is a $1 g_{9 / 2}$ proton decay of that level into the ground state of ${ }^{60} \mathrm{Zn}$. Using $E_{x}\left(9 / 2^{+}\right) \sim 2.4 \mathrm{MeV}$ together with the known binding energy one can estimate $Q_{p} \sim 2.2 \mathrm{MeV}$ for such a decay, which is very similar to the energetics of $1 g_{9 / 2}$ prompt proton decays from deformed to near spherical states in 
the mass region [1]. In the present case, however, no significant shape change should be associated with the decay, which further enhances its likelihood.

\section{SUMMARY AND OUTLOOK}

Nuclei in the vicinity of ${ }^{56} \mathrm{Ni}$ provide a plethora of nuclear structure phenomena. They allow for tests of isospin symmetry via the spectroscopy of mirror nuclei, and their 'complete' high-spin excitation schemes allow for thorough tests of both the spherical shell model and a variety of mean field models and, not least, their comparison and relation. Prompt particle decays are established decay modes from rotational structures in the second minimum of the nuclear potential into near spherical states in the first minimum of the nuclear potential. The study of this exotic decay mode requires new high-resolution charged particle detector systems coupled to the existing $\gamma$-ray spectrometers as well as other ancillary devices to perform combined in-beam $\gamma$-ray and charged-particle spectroscopy. From a technical aspect the limits of the particle energy resolution needs to be probed, not at least in view of future developments for highly pixelized charged-particle arrays for the next generation of $4 \pi \gamma$-ray spectrometers, namely GreTA and AGATA.

\section{ACKNOWLEDGEMENTS}

First of all, D.G. Sarantites and his co-workers from Washington University deserve credit for their perfect and persistent work and support concerning the preparation and conduction of the high-resolution Si-strip experiments. We would also like to thank all friends and colleagues who participated in one way or another in the experiments, in particular the local staff at both Argonne and Oak Ridge as well as the ATLAS and the HRIBF accelerator crews. This research was supported in part by the Swedish Research Council.

\section{REFERENCES}

1. D. Rudolph et al., Phys. Rev. Lett. 80, 3018 (1998).

2. D. Rudolph et al., Phys. Rev. Lett. 82, 3763 (1999).

3. D. Rudolph et al., Phys. Rev. Lett. 89, 022501 (2002).

4. D. Rudolph et al., Phys. Rev. Lett. 86, 1450 (2001).

5. D. Rudolph et al., Phys. Rev. C 63, 021301(R) (2001).

6. D.S. Delion, R.J. Liotta, and R. Wyss, Phys. Rev. C 68, 054603 (2003).

7. I.-Y. Lee, Nucl. Phys. A520, 641c (1990).

8. D. Rudolph et al., Eur. Phys. J. A 6, 377 (1999).

9. D. Rudolph et al., Eur. Phys. J. A 14, 137 (2002).

10. D. Rudolph et al., Nucl. Phys. A694, 132 (2001).

11. B. Davin et al., Nucl. Instrum. Meth. A473, 302 (2001).

12. D.G. Sarantites et al., Nucl. Instr. Meth. A381, 418 (1996).

13. D. Rudolph et al., to be published.

14. S.G. Kadmensky and A.A. Sonzogni, Phys. Rev. C 62, 054601 (2000).

15. S.G. Kadmensky, Phys. At. Nucl. 65, 831 (2002).

16. D.L. Hill and J.A. Wheeler, Phys. Rev. 89, 1102 (1953). 
17. N. Carjan, P. Talou, and D. Strottmann, in Proc. The Nucleus: New Physics for the New Millenium, Faure, South Africa, January 1999, Eds. F.D. Smit, R. Lindsay, and S.V. Förtsch, Kluwer Academic / Plenum Publishers, New York, 1999, p. 115.

18. P. Talou, in Proc. International Workshop Pingst $2000-$ Selected Topics on $N=Z$ Nuclei, June 2000, Lund, Sweden, Eds. D. Rudolph and M. Hellström, (Bloms i Lund AB, 2000), p. 10.

19. N.J. Stone and J. Rikovska, in Proc. Exotic Nuclei at the Proton Drip Line, Camerino, Italy, September 2001, Eds. C.M. Petrache and G. Lo Bianco, Universitá di Camerino, 2001, p. 229.

20. N.J. Stone, J. Rikovska, Sun Punan, and A. Woehr, in Proc. 12th International Conference on Hyperfine Interactions, Park City, Utah, August 2001, Eds. W. Evenson, H. Jaeger, and M.O. Zacate, Hyp. Int. 136, 143 (2001).

21. D.G. Sarantites et al., Nucl. Instr. Meth. A530, 473 (2004).

22. C.N. Davids et al., Nucl. Instr. Meth. B70, 358 (1992).

23. A. Jokinen et al., Eur. Phys. J. direct A 3, 1 (2002).

24. L.-L. Andersson et al., submitted to Phys. Rev. C.

25. I. Ragnarsson, priv. comm.

26. M.A. Bentley et al., in Proceedings Pingst 2000 - Selected Topics on $N=Z$ Nuclei, Eds. D. Rudolph and M. Hellström (Bloms i Lund, Sweden, 2000), p. 222.

27. S.M. Lenzi and P.G. Bizzeti, in Achievements with the EUROBALL spectrometer, Eds. W. Korten and S. Lunardi (2004), p. 59.

28. M.A. Bentley, C.D. O'Leary, A. Poves, G. Marínez-Pinedo, D.E. Appelbe, R.A. Bark, D.M. Cullen, S. Ertürk, and A. Maj, Phys. Lett. B 437, 243 (1998).

29. J. Ekman et al., Eur. Phys. J A 9, 13 (2000).

30. S.J. Williams et al., Phys. Rev. C 68, 011301(R) (2003).

31. S.M. Lenzi et al., Phys. Rev. Lett. 87, 122501 (2001).

32. P.E. Garrett et al., Phys. Rev. Lett. 87, 132502 (2001).

33. A.P. Zuker, S.M. Lenzi, G. Martínez-Pinedo, and A. Poves, Phys. Rev. Lett. 89, $142502(2002)$.

34. J. Ekman et al., Phys. Rev. Lett. 92, 132502, (2004).

35. C. J. Gross et al., Nucl. Instrum. \& Meth. in Phys. Res. A 450, 12-29 (2000).

36. L.-L. Andersson, Master Thesis, Lund University, LUNFD6/(NFFR-5022)1$47 /(2004)$.

37. S. M. Vincent et al., Phys. Rev. C 60, 064308 (1999).

38. O. Izotova et al., Phys. Rev. C 69, 037303 (2004).

39. M. R. Bhat, Nucl. Data Sheets 88, 417 (1999).

40. E.K. Johansson, Master Thesis, Lund University, LUNFD6/(NFFR-5023)1-47/(2004).

41. D. Rudolph et al., Phys. Rev. C 69, 034309 (2004).

42. J.Huo and B.Singh, Nucl. Data Sheets 91, 317 (2000).

43. J.A. Cameron and B. Singh, Nucl. Data Sheets 94, 429 (2001).

44. M. R. Bhat, Nucl. Data Sheets 85, 415 (1998).

45. J. Ekman et al., Phys. Rev. C, in press.

46. E. Caurier, shell model code ANTOINE, IRES, Strasbourg (1989-2002).

47. E. Caurier, F. Nowacki, Acta Phys. Pol. 30, 705, (1999). 